\title{
Pancreaticobiliary endoscopic ultrasound in England 2007 to 2016: Changing practice and outcomes
}

\section{다(1) $(9)$}

\author{
Authors \\ Nigel Trudgill ${ }^{*}, 1$, Kofi W. Oppong*,3,5 \\ Institutions \\ 1 Department of Gastroenterology, Sandwell and West \\ Birmingham Hospitals NHS Trust, United Kingdom \\ 2 Health Informatics, University Hospitals Birmingham \\ NHS Foundation Trust, United Kingdom \\ 3 HPB Unit and Department of Gastroenterology, \\ Newcastle upon Tyne Hospitals NHS Foundation Trust, \\ United Kingdom \\ 4 Population Health Sciences Institute, Newcastle \\ University, United Kingdom \\ 5 Institute of Translational and Clinical Research, \\ Newcastle University, United Kingdom
}

Umair Kamran', Dominic King' ${ }^{1}$, Amandeep Dosanjh², Ben Coupland ${ }^{2}$, John Leeds ${ }^{3,4}$, Manu Nayar ${ }^{3}$, Prashant Patel2

submitted 1.4 .2021

accepted after revision $\quad 14.6 .2021$

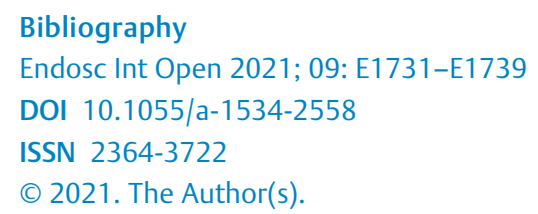

This is an open access article published by Thieme under the terms of the Creative Commons Attribution-NonDerivative-NonCommercial License, permitting copying and reproduction so long as the original work is given appropriate credit. Contents may not be used for commercial purposes, or adapted, remixed, transformed or built upon. (https://creativecommons.org/licenses/by-nc-nd/4.0/)

Georg Thieme Verlag KG, Rüdigerstraße 14,

70469 Stuttgart, Germany

Corresponding author

Dr. Kofi W. Oppong, HPB Unit and Department of Gastroenterology, Newcastle upon Tyne Hospitals NHS Foundation Trust, NE7 7DN, United Kingdom kofi.oppong@nhs.net

Supplementary material is available under https://doi.org/10.1055/a-1534-2558

\section{ABSTRACT}

Background and study aims Population-level data on the outcomes of pancreaticobiliary endoscopic ultrasound (PBEUS) are limited. We examined national PB-EUS and fineneedle aspiration (FNA) activity, its relation to pancreatic cancer therapy, associated mortality and adverse events.

Patients and methods Adults undergoing PB-EUS in England from 2007-2016 were identified in Hospital Episode Statistics. A pancreatic cancer cohort diagnosed within 6 months of PB-EUS were studied separately. Multivariable logistic regression models examined associations with 30-day mortality and therapies for pancreatic cancer.

Results 79,269 PB-EUS in 68,908 subjects were identified. Annual numbers increased from 2,874 (28\% FNA) to 12,752 (35\% FNA) from 2007 to $2016.8,840$ subjects (13\%) were diagnosed with pancreatic cancer. Sedation related adverse events were coded in $0.5 \%$ and emergency admission with acute pancreatitis in $0.2 \%$ within 48 hours of PB-EUS. $1.5 \%$ of subjects died within 30 days of PB-EUS. Factors associated with 30-day mortality included increasing age (odds ratio 1.03 [95\% Cl 1.03-1.04]); male sex (1.38 [1.24-1.56]); increasing comorbidity (1.49 [1.27-1.74]); EUS-FNA (2.26 [1.98-2.57]); pancreatic cancer (1.39 [1.19-1.62]); increasing deprivation (least deprived quintile 0.76 [0.620.93]) and lower provider PB-EUS volume (2.83 [2.15$3.73]$ ). Factors associated with surgical resection in the pancreatic cancer cohort included lower provider PB-EUS volume $(0.44$ [0.26-0.74]) and the least deprived subjects (1.33 [1.12-1.57]). $33 \%$ of pancreatic cancer subjects who underwent EUS, did not subsequently receive active cancer treatment.

Conclusions Lower provider PB-EUS volume was associated with higher 30-day mortality and reduced rates of both pancreatic cancer surgery and chemotherapy. These results suggest potential issues with case selection in lower-volume EUS providers.

\footnotetext{
* These authors contributed equally
} 


\section{Introduction}

Pancreaticobiliary endoscopic ultrasound (PB-EUS) has an established role in the assessment, staging, and sampling (via EUS-guided fine-needle aspiration [FNA] or biopsy [FNB]) of pancreaticobiliary disease, and increasingly, EUS-guided interventions such as the drainage of pancreatic fluid collections $[1,2]$. Over the last 10 years, EUS-FNA/FNB has become the standard modality for sampling solid and cystic pancreatic lesions. There is extensive literature on its diagnostic performance in solid and cystic disease of the pancreas [3-7]. The morbidity and mortality from EUS-FNA/FNB in solid pancreatic lesions is reported to be $2.4 \%$ and $0.02 \%$, respectively [8] and in cystic pancreatic lesions $2.7 \%$ and $0.2 \%$, respectively [9].

However, there are no large, long-term prospective studies of outcomes following PB-EUS and the impact of procedural and non-procedural factors. In the absence of such prospective data, population-level observational studies offer the potential to understand PB-EUS practice and the risk of adverse events associated with EUS and EUS-FNA. There have been few such studies to date. A recent US study utilizing the Surveillance, Epidemiology, and End Results (SEER) database addressed concerns about peritoneal seeding following EUS-FNA for pancreatic cancer and reported no adverse effect of prior EUS-FNA on pancreatic cancer survival [10]. EUS assessment has been reported to be an independent predictor of improved survival in locoregional pancreatic cancer through the improved stage-appropriate management consequent on EUS [10,11].

We have examined national changes in the use of EUS and EUS-FNA, to try and identify the factors contributing to adverse events (AEs) and 30-day mortality after EUS. We have separately studied subjects with pancreatic cancer who underwent PBEUS to understand the factors associated with surgical resection, chemotherapy, and not receiving any active treatment.

\section{Patients and methods}

\section{Data source}

Administrative data on all care episodes taking place in National Health Service (NHS) hospitals in England are recorded in the Hospital Episode Statistics (HES) database. Unique identifiers track subjects through inpatient and outpatient attendances. Every recorded episode contains clinical information about diagnoses and procedures, demographic, administrative, and geographical information. Diagnostic data are coded using the International Classification of Diseases version 10 (ICD-10) and procedures coded using the Office of Population Censuses and Surveys Classification of Interventions and Procedures $4^{\text {th }}$ revision (OPCS-4). HES is linked to the Office of National Statistics mortality data, providing information on the date and cause of death [12].

\section{Inclusion criteria}

All adults over age 18 years undergoing PB-EUS, both as inpatients and outpatients, in England over 10 years (2007-2016) were included in the study. Subjects diagnosed with pancreatic cancer by any means within the 6 months following PB-EUS were included in the PB-EUS pancreatic cancer cohort. Diagnostic data were obtained from ICD-10 coding and procedure data were obtained from OPCS-4 coding (Appendix 1).

\section{Exclusion criteria}

Subjects were excluded if they were under age 18 years, resided outside England, or had incomplete demographic data. EUS procedures for non-PB indications were also excluded from the study.

\section{Data validation}

To assess the validity of coding for PB-EUS, a list of subjects coded for all EUS, specifically PB-EUS and FNA procedures, was provided by the local coding departments at Sandwell and West Birmingham NHS Trust, Newcastle upon Tyne Hospitals NHS Foundations Trust and University Hospitals Birmingham NHS Foundation Trust. These data were used to match the number of PB-EUS procedures extracted from the HES database for each individual site. A sample of patient records from all three sites were analyzed by the research team to establish the accuracy of procedural coding only, but not any other clinical details, to establish if the procedure coding was consistent with the clinical records.

\section{Demographic data}

Demographic data including age, sex, ethnicity, and deprivation were extracted from hospital admission coding. Age was considered a continuous variable. Ethnicity was classified into White, South Asian, Black, mixed ethnicity, and other minority ethnicities. Deprivation was calculated using an aggregate score for English Lower Layer Super Output Areas, based on employment status, income, crime levels, and living environment [13]. Deprivation was categorized by quintiles, with 1 being the most deprived and 5 being the least deprived.

A modified Charlson comorbidity score was calculated using ICD-10 codes for secondary diagnoses, excluding any form of cancer or diabetes mellitus. The Charlson comorbidity score has previously been validated in HES [14].

\section{Healthcare providers}

PB-EUS providers were stratified based on their number of procedures over the 10 years study period. Centers with ultra-low volume of PB-EUS activity ( $<10$ procedures over the 10 -year study period) were excluded. Health care providers were grouped into tertiles and the range of the number of procedures in each tertile was the natural consequence of having an equal number of centers in each tertile.

\section{Outcome measures}

Outcome measures included 30-day all-cause mortality and emergency readmission into any hospital within 30 days of discharge following PB-EUS.AEs included bleeding, perforation, pancreatitis, and sedation-related. As it can be difficult to establish from the HES if pancreatitis was the indication or a complication of PB-EUS, if it was coded within the same admission episode, we decided to exclude the subjects from the analysis of AEs if they had ICD-10 codes for acute pancreatitis concur- 
rent with their PB-EUS and include the subjects as having pancreatitis only when they were readmitted with acute pancreatitis within 48 hours of PB-EUS. This methodology has a limitation that pancreatitis following an inpatient PB-EUS would not be captured. For all other complications, all complications within 7 days of post PB-EUS were included. Patients who had ERCP 7 days before or after EUS were excluded from analysis of these AEs to avoid difficulties with attributing the complication. Rates of surgical resection in pancreatic cancer patients, chemotherapy, and no active cancer treatment were also examined.

\section{Statistical analysis}

All statistical analyses were carried out using STATA SE v15. Categorical variables are presented as numbers and percentages (\%), while continuous variables are described as median (interquartile range). Multivariable logistic regression analysis of the factors associated with 30-day PB-EUS mortality was modeled adjusting for age, sex, deprivation quintile, ethnicity, modified Charlson score, EUS sampling with FNA/FNB, post-EUS diagnosis of pancreatic cancer, and provider volume of PB-EUS. Further logistic regression analysis was performed to identify factors associated with surgical resection, chemotherapy and no active therapy in the PB-EUS pancreatic cancer group. $P<0.05$ was considered statistically significant.

\section{Ethics}

HES data are available under a data sharing agreement with NHS Digital for the purposes of service evaluation and as such ethics approval is not required. Numbers less than 6 were censored from publication to protect subject anonymity. The study was registered at the University Hospitals Birmingham NHS Foundation Trust.

\section{Results}

\section{Data validation}

Sandwell and West Birmingham NHS Trust performed 344 PBEUS with a coding accuracy rate of $90.8 \%$. Newcastle upon Tyne Hospitals NHS Foundation Trust performed 6,444 PB-EUS with a coding accuracy rate of $92.9 \%$. University Hospitals Birmingham NHS Foundation Trust performed 6,672 PB-EUS with a coding accuracy rate of $97.4 \%$.

\section{Cohort characteristics}

During the study period (2007 to 2016), 79,269 PB-EUS procedures were performed in 68,908 subjects in England and $87 \%$ were index procedures (Appendix 2). The median age of subjects who underwent PB-EUS was 63 (IQR 51-72) years and 56 $\%$ were women. The majority of PB-EUS (82\%) were performed in higher-volume providers (defined as $>816$ over the study period).

Pancreatic cancer was diagnosed in $13 \%$ of patients within 6 months of index EUS. Demographic data for all subjects who underwent PB-EUS and the PB-EUS cohort with pancreatic cancer are presented in $\downarrow$ Table $\mathbf{1}$.
Eighty-seven percent of subjects had a single EUS within the 6 months prior to a diagnosis of pancreatic cancer, $11 \%$ had two, $2 \%$ had three, and 20 subjects had four EUS procedures prior to pancreatic cancer diagnosis.

\section{Changing PB-EUS and FNA practice}

The annual number of PB-EUS and the percentage with FNA performed consistently increased over the 10 years studied; 2874 PB-EUS (28\% FNA) in 2007 and 12,752 PB-EUS (35\% FNA) in 2016. EUS-FNA particularly increased in the PB-EUS pancreatic cancer group from $43 \%$ in 2007 to $78 \%$ in 2016 ( Fig. 1).

\section{Post PB-EUS adverse events}

The coded adverse events (AEs) following PB-EUS are shown in - Table 2. Sedation related events and acute pancreatitis were coded in $0.5 \%$ and $0.2 \%$ cases respectively. EUS-FNA was associated with a significantly higher rate of acute pancreatitis (0.4\% vs $0.09 \%)$.

\section{Post PB-EUS mortality}

A total of 1070 deaths (1.5\%) were reported within 30 days of index PB-EUS. Sixty- and 90-day mortality was reported in $3.7 \%$ and $5.5 \%$ of subjects. In the PB-EUS pancreatic cancer group, $2.8 \%$ of subjects died within 30 days of their EUS, $9 \%$ within 60 days and $15 \%$ within 90 days ( $>$ Table 3 ).

On multivariable analysis, increasing age, male sex, increasing comorbidities, and pancreatic cancer predicted higher mortality. Among procedural factors, FNA and lower-volume EUS providers were associated with higher mortality ( $\downarrow$ Table 4$)$.

\section{Factors associated with surgical resection, chemotherapy, and no active treatment in the PB-EUS pancreatic cancer cohort}

Twenty-four percent of patients in the PB-EUS pancreatic cancer cohort had a surgical resection: $12.4 \%$ had surgery alone; $0.7 \%$ received neoadjuvant chemotherapy; $10.3 \%$ had adjuvant chemotherapy; and $0.5 \%$ received both neoadjuvant and adjuvant chemotherapy. Forty-three percent of patients received chemotherapy alone and $33 \%$ of subjects did not receive any oncological or surgical treatment. Multivariable analysis of the variables associated with the likelihood of undergoing a surgical resection, chemotherapy alone, or no active treatment are presented in $>$ Table 5 .

Patients from less deprived areas were more likely to undergo surgical resection and chemotherapy while increasing age, increasing comorbidities (Charlson comorbidity score $>5$ ), and lower-volume PB-EUS providers were associated with lower rates of surgical resection and higher rates of no active treatment.

\section{Discussion}

The widespread adoption of EUS and EUS-guided tissue sampling has been a major advance in pancreatology over the last 20 years. This is the largest population-based study of patients who underwent PB-EUS. There was a more than four-fold increase in the annual number of PB-EUS procedures performed 
- Table 1 Baseline characteristics of subjects undergoing PB-EUS and the PB-EUS pancreatic cancer cohort.

\begin{tabular}{|c|c|c|c|}
\hline Parameters & & $\begin{array}{l}\text { All PB-EUS } \\
N(\%)\end{array}$ & $\begin{array}{l}\text { PB-EUS pancreatic cancer group } \\
\mathrm{N}(\%)\end{array}$ \\
\hline \multicolumn{2}{|l|}{ Total } & 79269 & 8840 \\
\hline \multirow[t]{2}{*}{ Age } & Median & 63 & 68 \\
\hline & $\mathrm{IQR}$ & $51-72$ & $60-75$ \\
\hline \multirow[t]{2}{*}{ Sex } & Male & $35052(44.2)$ & $4657(52.7)$ \\
\hline & Female & $44214(55.8)$ & $4183(47.3)$ \\
\hline \multirow[t]{6}{*}{ Deprivation $^{1}$} & 1 & $18033(22.7)$ & $1458(16.5)$ \\
\hline & 2 & $15017(18.9)$ & $1646(18.6)$ \\
\hline & 3 & $15701(19.8)$ & $1917(21.7)$ \\
\hline & 4 & 15741 (19.9) & $1998(22.6)$ \\
\hline & 5 & $14484(18.3)$ & $1818(20.6)$ \\
\hline & Unknown & $293(0.4)$ & $3(0.0)$ \\
\hline \multirow[t]{6}{*}{ Ethnicity } & White & $58921(74.3)$ & $8060(90.8)$ \\
\hline & Asian or Asian British & $2166(2.7)$ & $222(2.5)$ \\
\hline & Black or Black British & $1247(1.6)$ & $140(1.6)$ \\
\hline & Mixed & $347(0.4)$ & $40(0.5)$ \\
\hline & Any other ethnicity & $1327(1.7)$ & $169(1.9)$ \\
\hline & Unknown & $15260(16.8)$ & $208(2.1)$ \\
\hline \multirow[t]{3}{*}{ Charlson comorbidity score } & $<1$ & $60921(76.9)$ & $6758(76.4)$ \\
\hline & $1-5$ & $11968(15.1)$ & $1519(17.2)$ \\
\hline & 5 & $6380(8.0)$ & $563(6.4)$ \\
\hline \multicolumn{2}{|l|}{ Pancreatic cancer } & $10179(12.8)$ & $8840(100.0)$ \\
\hline \multirow[t]{3}{*}{ Provider volume of PB-EUS } & $10-163$ & $1,355(2.0)$ & \\
\hline & $164-816$ & $10,863(15.8)$ & \\
\hline & $>816$ & $56,690(82.3)$ & \\
\hline
\end{tabular}

during the study period. This is consistent with the previously published findings from the United States [15] and Canada [16] and reflects the increase in availability of EUS and its emerging role in pancreaticobiliary diseases. The proportion of subjects who were diagnosed with pancreatic cancer within 6 months of EUS remained fairly constant at $13 \%$. This is consistent with the fact that the incidence rates of pancreatic cancer in the UK have not changed over the past 10 years (European age standardized incidence rate per 100,000 population; 15.5 in 2007 vs 16.9 in 2016) [17].

EUS-guided tissue sampling has become the method of choice to investigate pancreatic mass lesions due to its high accuracy, with a sensitivity of $85 \%$ to $89 \%$ and specificity of $96 \%$ to $99 \%$ according to three meta-analyses $[4,18,19]$. Consequently, there was a substantial increase in the use of EUS-guid- ed sampling in the pancreatic cancer group over the course of the study period from $43 \%$ in 2007 to $78 \%$ in 2016 .

PB-EUS is considered a safe procedure with an overall AE rate estimated to be approximately $1 \%$ [20]. The main AEs include perforation, bleeding, pancreatitis, and those related to sedation. The longer rigid terminal end $(4-5 \mathrm{~cm})$ of the echoendoscope and semi-blind intubation and maneuvering may potentially increase the rate of perforation, compared with upper gastrointestinal endoscopy. In the present study, perforation was reported in $0.03 \%$ cases, which is comparable to the previously reported range of between $0.03 \%$ and $0.06 \%[21,22]$. Bleeding was reported in $0.05 \%$ cases, which is in line with reported incidence rates of $0 \%$ to $0.5 \%$ in large prospective series [23-27]. FNA sampling was not found to be associated with an increased risk of bleeding. Data on the severity or site of bleeding were unfortunately not available to investigate this further. 


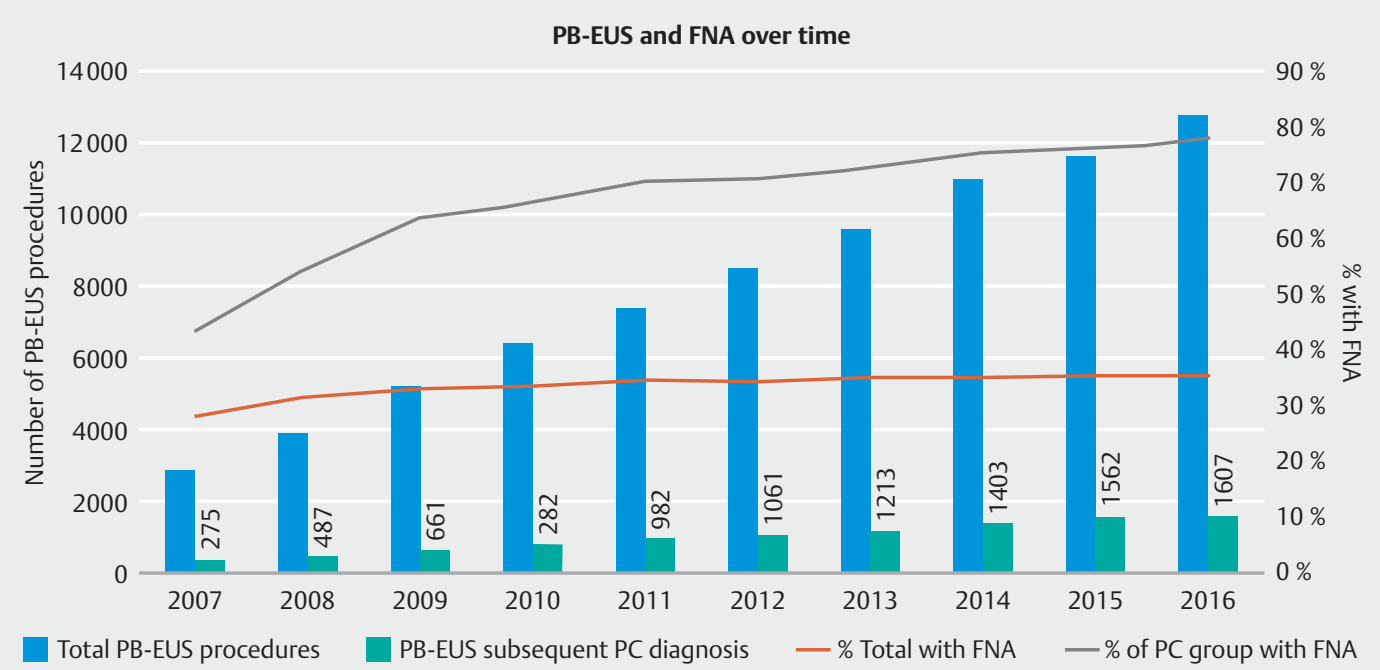

- Fig. 1 Changes in the annual number of pancreaticobiliary endoscopic ultrasound-guided fine-needle aspirations or biopsies in subjects with a subsequent pancreatic cancer diagnosis. PB-EUS, pancreaticobiliary endoscopic ultrasound; FNA, fine-needle aspiration or biopsy; PC, pancreatic cancer.

- Table 2 Post pancreaticobiliary endoscopic ultrasound adverse events.

\begin{tabular}{|c|c|c|c|c|}
\hline & $\begin{array}{l}\text { All } \\
\mathrm{N}(\%)\end{array}$ & $\begin{array}{l}\text { EUS-FNA } \\
\text { N (\%) }\end{array}$ & $\begin{array}{l}\text { No FNA } \\
\text { N (\%) }\end{array}$ & $P$ value \\
\hline Number of procedures & 79269 & 27239 & 52030 & \\
\hline Bleeding & $36(0.05)$ & $17(0.06)$ & $19(0.04)$ & 0.10 \\
\hline Perforation & $27(0.03)$ & $13(0.04)$ & $14(0.02)$ & 0.19 \\
\hline Pancreatitis & $160(0.2)$ & $114(0.42)$ & $46(0.09)$ & $<0.001$ \\
\hline Sedation related & $370(0.5)$ & $139(0.5)$ & $231(0.4)$ & 0.21 \\
\hline
\end{tabular}

PB-EUS, pancreaticobiliary endoscopic ultrasound; EUS-FNA, endoscopic ultrasound-guided fine-needle aspiration or biopsy.

- Table 3 Mortality following pancreaticobiliary endoscopic ultrasound.

\begin{tabular}{|l|l|l|l|l|l|l|}
\hline & \multicolumn{2}{|l|}{ All PB-EUS patients } & \multicolumn{2}{|l|}{ PB-EUS pancreatic cancer cohort } \\
\hline & All & EUS-FNA & $\begin{array}{l}\text { Multiple EUS between } \\
\text { index and death } \\
\text { N (\%) }\end{array}$ & $\begin{array}{l}\text { All subjects } \\
\text { N (\%) }\end{array}$ & $\begin{array}{l}\text { EUS-FNA } \\
\text { N (\%) }\end{array}$ & $\begin{array}{l}\text { Multiple EUS between } \\
\text { index and death } \\
\text { N(\%) }\end{array}$ \\
\hline $\begin{array}{l}\text { Total number of } \\
\text { subjects }\end{array}$ & 68908 & 24719 & 1599 & 8540 & 521 \\
\hline 30-day mortality & $1070(1.5)$ & $634(2.6)$ & $46(2.9)$ & $251(2.8)$ & $182(2.8)$ & $16(3.1)$ \\
\hline 60-day mortality & $2525(3.7)$ & $1633(6.6)$ & $99(6.2)$ & $792(9)$ & $584(8.9)$ & $37(7.1)$ \\
\hline 90-day mortality & $3821(5.5)$ & $2490(10)$ & $155(9.7)$ & $1329(15)$ & $996(15.1)$ & $66(12.7)$ \\
\hline PB-EUS, pancreaticobiliary endoscopic ultrasound; EUS-FNA, endoscopic ultrasound-guided fine-needle aspiration or biopsy.
\end{tabular}

Confounding due to bleeding related to the underlying pathology being investigated by PB-EUS remains possible. Acute pancreatitis was reported in $0.2 \%$ cases and its incidence was significantly higher following FNA. Similar rates of pancreatitis were reported in a multicenter survey but it was noted that the frequency of post-EUS pancreatitis may be underestimated by retrospective analyses [28]. In a prospective study of 100 consecutive patients, Gress et al. reported clinical and biochemical incidence of pancreatitis in two patients (2\%), who both responded to conservative management [29]. The asso- 
- Table4 Multivariable analysis of factors associated with 30-day post pancreaticobiliary endoscopic ultrasound mortality.

\begin{tabular}{|c|c|c|c|c|}
\hline \multicolumn{2}{|l|}{ Parameters } & Odds Ratio & $95 \% \mathrm{Cl}$ & $P$ value \\
\hline \multicolumn{2}{|l|}{ Age } & 1.03 & $1.03-1.04$ & $<0.001$ \\
\hline \multirow[t]{2}{*}{ Sex } & Female & \multicolumn{3}{|l|}{ Reference } \\
\hline & Male & 1.38 & $1.24-1.56$ & $<0.001$ \\
\hline \multirow[t]{6}{*}{ Deprivation $^{1}$} & 1 & \multicolumn{3}{|l|}{ Reference } \\
\hline & 2 & 0.93 & $0.78-1.13$ & 0.471 \\
\hline & 3 & 0.87 & $0.72-1.04$ & 0.130 \\
\hline & 4 & 0.82 & $0.68-0.99$ & 0.038 \\
\hline & 5 & 0.76 & $0.62-0.93$ & 0.007 \\
\hline & Unknown & 0.34 & $0.05-2.44$ & 0.281 \\
\hline \multirow[t]{6}{*}{ Ethnic group } & White & \multicolumn{3}{|l|}{ Reference } \\
\hline & Asian or Asian British & 1.20 & $0.85-1.70$ & 0.307 \\
\hline & Black or Black British & 0.67 & $0.38-1.20$ & 0.178 \\
\hline & Mixed & 0.62 & $0.15-2.53$ & 0.509 \\
\hline & Any Other Ethnicity & 0.94 & $0.58-1.54$ & 0.817 \\
\hline & Unknown & 1.08 & $0.91-1.29$ & 0.352 \\
\hline \multirow[t]{3}{*}{ Charlson comorbidity score } & $<1$ & \multicolumn{3}{|l|}{ Reference } \\
\hline & $1-5$ & 1.49 & $1.27-1.74$ & $<0.001$ \\
\hline & $>5$ & 2.99 & $2.56-3.50$ & $<0.001$ \\
\hline \multicolumn{2}{|l|}{ EUS-FNA } & 2.26 & $1.98-2.57$ & $<0.001$ \\
\hline \multicolumn{2}{|l|}{ Pancreatic cancer } & 1.39 & $1.19-1.62$ & $<0.001$ \\
\hline \multirow[t]{3}{*}{ Provider volume of PB- EUS } & $>816$ & \multicolumn{3}{|l|}{ Reference } \\
\hline & $164-816$ & 1.20 & $1.02-1.41$ & 0.025 \\
\hline & $10-163$ & 2.83 & $2.15-3.73$ & $<0.001$ \\
\hline
\end{tabular}

ciation of bleeding and pancreatitis with EUS without FNA is unexpected and it is important to recognize that this may relate to residual confounding due to AEs related to other concurrent procedures such as percutaneous transhepatic cholangiography, which were not excluded, or the underlying pathology being investigated by PB-EUS. The results of the present study indicate that PB-EUS is a safe procedure with overall a less than $1 \%$ risk of $A E s$.

Data on the factors associated with early post PB-EUS mortality are scarce. In a systematic review of more than 10,000 patients who underwent EUS-FNA, $0.02 \%$ mortality rate was reported to be attributable to procedure-related complications [8]. In another systematic review and meta-analysis of morbidity and mortality of EUS-FNA for cystic lesions, Zhu et al. reported a mortality rate of $0.19 \%$ ( $95 \% \mathrm{Cl} 0.09-0.32 \%$ ) [9]. However, non-procedural factors e.g comorbidities and variation between different providers were not studied, which are potentially important confounders of post-EUS outcomes. In the present study, 30-day post-PB-EUS all-cause mortality was recorded in $1.5 \%$ of all patients and $2.8 \%$ of PB-EUS patients with pancreatic cancer. On multivariable analysis, increasing age, male sex, and increasing comorbidity were associated with 30-day mortality. Among procedure-related factors, lower-volume providers and EUS-FNA were associated with higher mortality. Although EUS-FNA is reported to be safe with a low risk of morbidity and mortality [9], it may be a surrogate for significant pancreaticobiliary pathology, which may explain the association with mortality. Causes of deaths were not analyzed and it is possible that fatal complications of other invasive procedures such as surgery within 30 days of PB-EUS might have contributed in a small number of cases.

Pancreatic cancer is the fifth most common cause of cancer deaths in UK with a 1 -year survival rate of only $25 \%$ [30]. Thirteen percent of patients were diagnosed with pancreatic cancer within 6 months of the index EUS. While suspected pancreatic cancer is an important indication for PB-EUS, the majority of 
- Table 5 Multivariable analysis of factors associated with the likelihood of surgical resection, chemotherapy alone and no active therapy in the pancreaticobiliary endoscopic ultrasound pancreatic cancer cohort.

\begin{tabular}{|c|c|c|c|c|c|c|c|}
\hline \multirow{2}{*}{\multicolumn{2}{|c|}{ Variables }} & \multicolumn{2}{|l|}{$\begin{array}{l}\text { Surgical resection } \\
N=2111(24 \%)\end{array}$} & \multicolumn{2}{|c|}{$\begin{array}{l}\text { Chemotherapy alone } \\
\mathrm{N}=3809 \text { (43\%) }\end{array}$} & \multicolumn{2}{|l|}{$\begin{array}{l}\text { No active therapy } \\
\mathrm{N}=2920 \text { (33\%) }\end{array}$} \\
\hline & & OR (95\%Cl) & $P$ value & OR (95\%Cl) & $P$ value & O R (95\%Cl) & $P$ value \\
\hline \multicolumn{2}{|l|}{ Age } & $0.97(0.96-0.97)$ & $<0.001$ & $0.99(0.99-1.00)$ & $<0.001$ & $1.04(1.04-1.05)$ & $<0.001$ \\
\hline \multirow[t]{2}{*}{ Gender } & Male & \multicolumn{6}{|l|}{ Reference } \\
\hline & Female & $1.07(0.97-1.19)$ & 0.164 & $0.95(0.88-1.04)$ & 0.27 & $0.99(0.90-1.09)$ & 0.85 \\
\hline \multirow[t]{5}{*}{ Deprivation ${ }^{1}$} & 1 & \multicolumn{6}{|l|}{ Reference } \\
\hline & 2 & $1.09(0.92-1.29)$ & 0.340 & $1.20(1.04-1.39)$ & 0.012 & $0.76(0.65-0.88)$ & $<0.001$ \\
\hline & 3 & $1.12(0.95-1.33)$ & 0.177 & $1.26(1.10-1.45)$ & 0.001 & $0.70(0.60-0.81)$ & $<0.001$ \\
\hline & 4 & $1.31(1.11-1.55)$ & 0.001 & $1.20(1.04-1.38)$ & 0.012 & $0.65(0.56-0.75)$ & $<0.001$ \\
\hline & 5 & $1.33(1.12-1.57)$ & 0.001 & $1.14(0.99-1.31)$ & 0.076 & $0.68(0.58-0.79)$ & $<0.001$ \\
\hline \multirow[t]{6}{*}{ Ethnic group } & White & \multicolumn{6}{|l|}{ Reference } \\
\hline & Asian or Asian British & $1.01(0.74-1.38)$ & 0.955 & $0.92(0.70-1.21)$ & 0.550 & $1.07(0.80-1.45)$ & 0.642 \\
\hline & Black or Black British & $0.72(0.46-1.11)$ & 0.136 & $0.98(0.70-1.38)$ & 0.920 & $1.29(0.90-1.85)$ & 0.167 \\
\hline & Mixed & $0.76(0.36-1.60)$ & 0.471 & $1.04(0.55-1.95)$ & 0.901 & $1.12(0.55-2.28)$ & 0.747 \\
\hline & Any Other Ethnicity & $1.07(0.74-1.55)$ & 0.709 & $1.07(0.79-1.46)$ & 0.673 & $0.87(0.62-1.23)$ & 0.436 \\
\hline & Unknown & $0.48(0.32-0.72)$ & $<0.001$ & $0.96(0.72-1.27)$ & 0.765 & $1.72(1.29-2.28)$ & $<0.001$ \\
\hline \multirow{3}{*}{$\begin{array}{l}\text { Charlson } \\
\text { comorbidity } \\
\text { score }\end{array}$} & $<1$ & \multicolumn{6}{|l|}{ Reference } \\
\hline & $1-5$ & $0.96(0.84-1.10)$ & 0.530 & $0.89(0.79-0.99)$ & 0.037 & $1.19(1.06-1.35)$ & 0.004 \\
\hline & $>5$ & $0.71(0.57-0.90)$ & 0.004 & $0.65(0.54-0.78)$ & $<0.001$ & $1.93(1.61-2.30)$ & $<0.001$ \\
\hline \multirow{2}{*}{$\begin{array}{l}\text { Stent before } \\
\text { EUS }\end{array}$} & Metal & $1.28(0.90-1.81)$ & 0.169 & $0.99(0.73-1.35)$ & 0.969 & $0.82(0.58-1.15)$ & 0.241 \\
\hline & Plastic & $1.14(0.98-1.32)$ & 0.096 & $0.94(0.83-1.08)$ & 0.394 & $0.97(0.84-1.12)$ & 0.654 \\
\hline \multicolumn{2}{|c|}{ Multiple EUS pre-diagnosis } & $0.80(0.66-0.96)$ & 0.017 & $1.14(0.98-1.33)$ & 0.087 & $1.03(0.88-1.22)$ & 0.697 \\
\hline \multirow{3}{*}{$\begin{array}{l}\text { Provider } \\
\text { volume of } \\
\text { PB- EUS }\end{array}$} & $>816$ & \multicolumn{6}{|l|}{ Reference } \\
\hline & $164-816$ & $0.65(0.56-0.75)$ & 0.000 & $1.24(1.11-1.39)$ & $<0.001$ & $1.08(0.96-1.22)$ & 0.185 \\
\hline & $10-163$ & $0.44(0.26-0.74)$ & 0.002 & $1.07(0.76-1.51)$ & 0.699 & $1.56(1.10-2.22)$ & 0.013 \\
\hline
\end{tabular}

patients undergo the procedure for a benign indication, such as investigation of pancreatic cysts, unexplained pancreatitis, biliary type pain, unexplained dilated common bile duct, and for therapeutic intervention. It is not possible to determine the indication for each EUS from HES; however, a study published from an English cancer center in the early part of this study period reported on an annual case load approaching 700 EUS per year, of which, $5 \%$ were for drainage procedures, $29 \%$ EUSFNA for suspected malignancy and cysts, and $66 \%$ for a benign indication [31]. Surgical resection is the only curative therapy but most of the patients are diagnosed at an advanced stage, which precludes curative treatment. Twenty-four percent of patients with pancreatic cancer studied had a surgical resection, which is higher than the $10 \%$ of patients undergoing surgical resection for pancreatic cancer in the UK [32]. This differ- ence likely reflects selection bias. In a population-based study of pancreatic cancer patients, utilizing the SEER database, Ngamruengphong et al. also reported that patients who underwent EUS were more frequently offered curative treatment than those who did not and they were diagnosed at an earlier stage [11].

Patients who underwent PB-EUS through lower-volume providers were less likely to receive surgical treatment. This difference may at least in part be due to patients considered more likely to be suitable for surgery being transferred to higher-volume centers for multidisciplinary management including EUS. The presence of metallic or plastic stents prior to PB-EUS did not impact the likelihood of surgical treatment. It was noted that $33 \%$ of patients with pancreatic cancer did not receive any specific treatment, probably due to advanced disease at 
the time of diagnosis. The need for a definitive tissue diagnosis to exclude other treatable disease such as lymphoma or autoimmune diseases may account for some of these cases. No active treatment was associated with lower-volume PB-EUS providers as was a higher 30-day mortality rate, possibly indicating better case selection with higher-volume providers. In England regional specialized pancreatic cancer centers (where all surgical resections are performed) are generally the higher-volume EUS providers. The differences associated with provider volume may relate to multidisciplinary team discussion and planning reducing the use of EUS in patients unlikely to benefit. The link between deprivation and reduced surgical resection identified in this study is consistent with previous findings for all cancers [33] and pancreatic cancer specifically [34].

Although large observational studies are powerful tools for identifying risks and associations within populations, there are a number of limitations to the present study. There is a potential for recording bias as the accuracy of coded data depends on the quality of the medical records and on the staff coding the records. However, in the 2012/2013 annual report on the quality of HES records, $99.3 \%$ of primary diagnoses and $99.9 \%$ of primary procedure codes were accurate [35]. Validation of EUS codes in our study revealed that coding accuracy was above 90 $\%$. Unfortunately, important data such as smoking, body mass index, performance status, and pancreatic cancer staging details were unavailable in the HES dataset. Important procedure-related details such as the procedural experience of the endoscopist and whether any technical difficulties were encountered are also not recorded in HES. Detailed data on the causes of deaths were not available, hence it was not possible to definitively explain the relatively high 90-day mortality in all patients undergoing PB-EUS (5.5\%). However, in addition to pancreatic cancer, underlying comorbidities and non-pancreatic cancer pancreaticobiliary pathology e.g cholangiocarcinoma, large pancreatic pseudocysts, and necrotizing pancreatitis are all associated with higher mortality and likely to have contributed to the mortality figures. Finally, the link between a pancreatic cancer diagnosis and PB-EUS is through coding records rather than directly from cancer registry data. It is not possible to ascertain in HES if a pancreatic cancer diagnosis was based on the histology obtained from EUS and this study relied instead on the temporal association between pancreatic cancer diagnosis and PB-EUS procedural coding.

\section{Conclusions}

PB-EUS activity significantly increased in England from 2007 to 2016 with an increasing proportion of FNA in patients with pancreatic cancer. A significant proportion of patients who underwent EUS and had a diagnosis of pancreatic cancer did not undergo surgery or receive chemotherapy. Deprivation and lower provider PB-EUS volume were associated with increased 30-day mortality rates and reduced rates of surgical resection for pancreatic cancer. EUS and tissue sampling should not be performed unless they will clearly influence decision regarding surgery or chemotherapy.

\section{Competing interests}

The authors declare that they have no conflict of interest.

References

[1] Oppong K. Advancing the diagnostic and therapeutic role of EUS in pancreaticobiliary disease: Hopkins Lecture 2016. Frontline Gastroenterol 2017; 8: 115-118

[2] De Lisi S, Giovannini M. Endoscopic ultrasonography: Transition towards the future of gastro-intestinal diseases. World J Gastroenterol 2016; 22: 1779-1786

[3] Nayar MK, Paranandi B, Dawwas MF et al. Comparison of the diagnostic performance of 2 core biopsy needles for EUS-guided tissue acquisition from solid pancreatic lesions. Gastrointest Endosc 2017; 85: 1017-1024

[4] Hewitt MJ, McPhail MJW, Possamai L et al. EUS-guided FNA for diagnosis of solid pancreatic neoplasms: a meta-analysis. Gastrointest Endosc 2012; 75: 319-331

[5] Oppong KW, Dawwas MF, Charnley RM et al. EUS and EUS-FNA diagnosis of suspected pancreatic cystic neoplasms: Is the sum of the parts greater than the CEA? Pancreatology 2015; 15: 531-537

[6] Cizginer S, Turner BG, Bilge AR et al. Cyst fluid carcinoembryonic antigen is an accurate diagnostic marker of pancreatic mucinous cysts. Pancreas 2011; 40: 1024-1028

[7] Thornton GD, McPhail MJW, Nayagam S et al. Endoscopic ultrasound guided fine needle aspiration for the diagnosis of pancreatic cystic neoplasms: A meta-analysis. Pancreatology 2013; 13: 48-57

[8] Wang K-X, Ben Q-W, Jin Z-D et al. Assessment of morbidity and mortality associated with EUS-guided FNA: a systematic review. Gastrointest Endosc 2011; 73: 283-290

[9] Zhu H, Jiang F, Zhu J et al. Assessment of morbidity and mortality associated with endoscopic ultrasound-guided fine-needle aspiration for pancreatic cystic lesions: A systematic review and meta-analysis. Dig Endosc 2017; 29: 667-675

[10] Ngamruengphong S, Swanson KM, Shah ND et al. Preoperative endoscopic ultrasound-guided fine needle aspiration does not impair survival of patients with resected pancreatic cancer. Gut 2015; 64: $1105-1110$

[11] Ngamruengphong S, Li F, Zhou Y et al. EUS and survival in patients with pancreatic cancer: a population-based study. Gastrointest Endosc 2010; 72: 78-83

[12] Hospital Episode Statistics. http://www.hscic.gov.uk/hes

[13] Index of Multiple Deprivation (IMD). 2007: https://data.gov.uk/dataset/bdc1e1a5-aaf3-4f5a-9988-82a11e341eb8/index-of-multiple-deprivation-imd-2007

[14] Nuttall M, van der Meulen J, Emberton M. Charlson scores based on ICD-10 administrative data were valid in assessing comorbidity in patients undergoing urological cancer surgery. J Clin Epidemiol 2006; 59: $265-273$

[15] D’Souza SL, Holub JL, Pavic BT et al. Multicenter evaluation of the utilization of endoscopic ultrasound. Dig Endosc 2016; 28: 738-743

[16] James PD, Hegagi M, Antonova L et al. Regional differences in use of endoscopic ultrasonography in Ontario: a population-based retrospective cohort study. CMAJ Open 2017; 5: 437-443

[17] Pancreatic cancer incidence statistics. 2015: https://www.cancerresearchuk.org/health-professional/cancer-statistics/statistics-by-cancer-type/pancreatic-cancer/incidence

[18] Hébert-Magee S, Bae S, Varadarajulu S et al. The presence of a cytopathologist increases the diagnostic accuracy of endoscopic ultra- 
sound-guided fine needle aspiration cytology for pancreatic adenocarcinoma: a meta-analysis. Cytopathology 2013; 24: 159-171

[19] Puli SR, Bechtold ML, Eloubeidi MA. how good is eus-fna in diagnosing the correct etiology for a solid pancreatic mass? A meta-analysis and systematic review. Gastrointest Endosc 2009; 69: 243

[20] Polkowski M, Larghi A, Weynand B et al. Learning, techniques, and complications of endoscopic ultrasound (EUS)-guided sampling in gastroenterology: European Society of Gastrointestinal Endoscopy (ESGE) Technical Guideline. Endoscopy 2012; 44: 190-206

[21] Eloubeidi MA, Tamhane A, Lopes TL et al. Cervical esophageal perforations at the time of endoscopic ultrasound: a prospective evaluation of frequency, outcomes, and patient management. Am J Gastroenterol 2009; 104: 53-56

[22] Das A, Sivak MV Jr, Chak A. Cervical esophageal perforation during EUS: a national survey. Gastrointest Endosc 2001; 53: 599-602

[23] Wiersema M, Vilmann P, Giovannini M et al. Prospective multicenter evaluation of eus guided fine needle aspiration biopsy (FNA): Diagnostic accuracy and complication assessment. Gastrointest Endosc 1996; 43: 432

[24] Mortensen MB, Fristrup C, Holm FS et al. Prospective evaluation of patient tolerability, satisfaction with patient information, and complications in endoscopic ultrasonography. Endoscopy 2005; 37: 146 153

[25] Al-Haddad M, Wallace M, Woodward T et al. The safety of fine-needle aspiration guided by endoscopic ultrasound: a prospective study. Endoscopy 2007; 40: 204-208

[26] Bournet B, Migueres I, Delacroix M et al. Early morbidity of endoscopic ultrasound: 13 years' experience at a referral center. Endoscopy 2006; 38: 349-354
[27] Eloubeidi MA, Tamhane A, Varadarajulu S et al. Frequency of major complications after EUS-guided FNA of solid pancreatic masses: a prospective evaluation. Gastrointest Endosc 2006; 63: 622-629

[28] Eloubeidi MA, Gress FG, Savides T] et al. Acute pancreatitis after EUSguided FNA of solid pancreatic masses: a pooled analysis from EUS centers in the United States. Gastrointest Endosc 2004; 60: 385-389

[29] Gress F, Michael H, Gelrud D et al. EUS-guided fine-needle aspiration of the pancreas: Evaluation of pancreatitis as a complication. Gastrointest Endosc 2002; 56: 864-867

[30] Pancreatic cancer survival statistics. 2015: https://www.cancerresearchuk.org/health-professional/cancer-statistics/statistics-by-cancer-type/pancreatic-cancer/survival

[31] Oppong KW, Richardson DL, Charnley RM et al. The development and evolution of a tertiary pancreaticobiliary endoscopic ultrasound service: lessons learned. Frontline Gastroenterol 2011; 2: 66-70

[32] Survival for all stages of pancreatic cancer. https://www.cancerresearchuk.org/about-cancer/pancreatic-cancer/survival

[33] Public Health England. Seven things we learned from our latest cancer treatment data. 2018: https://publichealthmatters.blog.gov.uk/ 2018/07/17/seven-things-we-learned-from-our-latest-cancer-treatment-data/

[34] Shapiro M, Chen Q, Huang Q et al. Associations of socioeconomic variables with resection, stage, and survival in patients with earlystage pancreatic cancer. JAMA Surgery 2016; 151: 338-345

[35] Health and Social Care Information Centre. The quality of nationally submitted health and social care data England, annual report, 2013, experimental statistics. https://digital.nhs.uk/data-and-information/ publications/statistical/the-quality-of-nationally-submitted-healthand-social-care-data/the-quality-of-nationally-submitted-healthand-social-care-data-england-2013-second-annual-report-experimental-statistics 